\title{
A Narrative Architecture for Story-Driven Location-Based Mobile Games
}

\author{
Katsiaryna Naliuka, Tara Carrigy, Natasa Paterson, and Mads Haahr \\ School of Computer Science and Statistics, Trinity College Dublin, Ireland
}

\begin{abstract}
Dramatic improvements in smartphones over the last few years have positioned them as a major platform for interactive media content. In addition to being much more portable than laptop computers, smartphones also support a sophisticated combination of GPS, sensors and communications interfaces that allow extracting context information related to their environment, such as location, orientation and weather data. This combination of mobility and context-sensitivity opens up interesting possibilities in relation to interactive narrative, and for example allows audience immersion into an interactive story to be improved by placing the content in physical locations that are of direct relevance to the story. In this paper, we present a general-purpose narrative architecture that allows a considerable range of story-based game and guide content to be expressed in location-aware manner. We also present a case study of an actual location-aware augmented reality game, which demonstrates the architecture in a commercial setting and shows that it is sufficiently lightweight to run on the current generation of smartphones.
\end{abstract}

Keywords: Narrative Architecture, Mobile Gaming and Storytelling

\section{Introduction}

Smartphones are emerging as a personal computational platform of significant popularity. The latest generation of handsets are equipped with a sophisticated combination of camera, GPS, orientation sensors and excellent data transmission capabilities. For the purposes of games, these features allow a much greater degree of sophistication than past generations of mobile games. In particular, they facilitate creation of a new genre: location-based mobile games (LBMGs).

One of the advantages of location-awareness is that it lends itself well to presenting the historical information by placing the player in a relevant context. This is why location-aware games have significant educational potential and can be used as interactive mobile guides. However, a significant challenge remains with regard to structuring the story content in a fashion that allows it to be presented adaptively in response to changes in user location and other types of context. To address this challenge, we have developed a flexible narrative architecture that allows non-linear stories with multiple branches to be delivered in a location-aware manner. In addition, the architecture supports efficient resource management, which is critical for mobile applications. 
In this paper we present the architecture and discuss our preliminary experiences with it in the context of Viking Ghost Hunt (VGH), a location-based, story-driven, augmented reality game that runs on Android smartphones. The game is a hybrid of a mystery game and Gothic ghost story that incorporates historical information about the Viking presence in Dublin (800-1169 AD). The locations, in which the game is played, are selected because of the association with the Irish Viking history and are of direct historical significance to the story.

The rest of the paper is structured as follows. In Sec. 2 we briefly review the related work. Sec. 3 describes Viking Ghost Hunt and the requirements it sets for the narrative architecture. Sec. 4 presents the architecture to support interactive context-dependent narrative, which is the main contribution of the paper. In Sec. 5 we describe how the architecture is applied in VGH. Sec. 6 contains discussion of the approach. Concluding remarks in Sec. 7 end the paper.

\section{Related work}

The capabilities of GPS enabled devices have motivated the development of numerous applications that use the information about the physical location of the user. Location-based mobile games (LBMGs) and tourist guides are popular applications for such location-aware technology. During the last few years a broad range of such applications was developed, ranging from virtual tourist guides [17] to location-aware multimedia storytelling [15]. The settings, in which locationaware applications are employed, range from the user strolling freely in the urban or countryside environment [16] to the user being a passenger on a journey, upon which he or she exercises no control [5].

Generally speaking location-aware applications can be more or less interactive, ranging from more traditional mobile guides with limited user interaction, such as the Lancaster GUIDE project [6] to, at the other extreme, highly interactive LBMGs [3]. Many of the latter operate in an open-ended game world with set up rules but with no underlying narrative, such as the Can you see me now? game where physical players chase the online users through the city streets. More sophisticated game world is created in the Pirates! [4] game, where players can undertake predefined missions, however the core of the game is interaction with other players and with NPCs.

The interaction in the game does not need to be limited to the explicit actions of the player. The player's behavior, such as walking in a particular direction or staying for some time in the particular place, can also be used to affect narrative. For instance, in the Geist project [12] the player walks through a historical site and is presented with location related stories. However, in contrast with conventional mobile guides where the user is always presented with the same content at the same location, in Geist the story is automatically adapted to the user experience. The player gets a different story depending on the order in which the locations are visited and on the amount of time spent in each location. Yet the application aims to bind these stories together in a consistent experience. It is worth noting that the boundary between LBMGs and interactive guides seems 
to be blurring, since factually correct information is incorporated in some games, and mobile guides aim to present the historical information to the user in more entertaining way. For example, the Who Killed Hanne Holmgaard? project [16] engages the player in a detective game, while at the same time familiarizing him or her with the history of Denmark under the Nazi occupation. From the other hand, in the Voices of Oakland project [7] the guided tour is shaped as engaging encounters with ghosts. In the classification of interactive mobile guides by Kjeldskov and Paay [10] LBMGs are included in this definition.

In our research we were concerned with developing an architecture for handling narrative suitable for a wide range of location-based and, more broadly, context-aware games. The beat sequencer from Façade is the closest example of similar architecture [13]. In Façade the story is told through beats, i.e., pieces of narrative united by a common topic. Beats are sequenced in response to player interaction. When one beat is over, the beat sequencer is responsible for selecting the next. The choice is guided by metadata associated with the beats, and each beat is characterized by its set of preconditions, priority and story effects. The goal of the beat sequencer is to select an available beat with the story tension effects matching an author-specified story tension arc.

\section{$3 \quad$ Viking Ghost Hunt}

The game that we constructed, Viking Ghost Hunt (VGH), is a location-based adventure game, driven by a Gothic ghost story set in Viking Dublin (800-1169 A.D.). In this game the player assumes the role of a paranormal investigator and moves around the city hunting for ghosts, collecting evidence and solving the mysteries of haunted Viking Dublin. The game is narrative-driven in the sense that, in order to progress the game, the player must unlock a sequence of distributed, location-specific, narrative fragments, which together make up a meaningful story arc. Hence, the VGH narrative, which is mapped onto actions and space, is revealed through the players' activities as they move through the locations and, in order to preserve the aesthetics of the role-play, these activities are presented in the context of paranormal investigation. In this form of storytelling, the player is not an external observer, but becomes an active player, who advances in the game and progresses the story by interacting with narrative elements, such as location, character and plotline.

As is typical of the quest structure, and common in the adventure game genre, the player's actions are crucial to accessing the narrative and the challenges faced by the player integrate the story elements into the game play [1, $8,9]$. The purpose of the quest, therefore, is two-fold; it is both symbolic and structural. By linking the narrative to the player's activities, the quest provides the player with meaningful goals while simultaneously functioning as a structure for achieving these goals through specific activities [19]. The quest provides a bridge between the game fiction and game rules and for this reason it is a useful technique for creating the illusion of agency, which Janet Murray describes as "the satisfying power to take meaningful action and see the results of our de- 
cisions and choices" [14, p.126]. Supported by storytelling it can be a powerful medium to convey the historical information to the player in entertaining form.

The characteristic of the quest structure that contributes most significantly to the player's sense of agency is the balance between embedded and emergent narrative $[11,18]$. Embedded narrative is pre-generated prior to player's interaction and tends to be more linear in nature. It contains the over-arching story arc and gives structure to the order in which the narrative is conveyed, thus preserving a coherent expression of the plotline. Emergent narrative is more open-ended and less predictable but is more responsive to the player's interaction and therefore facilitates a greater sense of agency. While a linear narrative structure fits easily with the classic Hollywood 3-act structure, which is popular for building dramatic tension and emotional impact, a non-linear, branching structure affords the player increased agency and the potential of re-playing the game.

With these considerations in mind, we were motivated to create a quest structure that could balance both embedded and emergent narrative. Multiple branching options were introduced at key points of an essentially linear structure, thus forming parallel story paths. In order to contain the potential unwieldiness of these optional branches, a set of inevitable event points to which all player must return, was introduced. This fold back structure [2], is especially useful in narrative-driven location-based gaming where game designers may also want to guide people through both essential narrative plot-points and specific locations.

The main success criteria for the architecture to support the described type of narrative can therefore be summarized as follows:

- it should be capable of supporting location-specific narrative;

- it should have ability to handle non-linear branching stories;

- as smartphones remain a resource-constrained platform it should facilitate efficient resource management.

\section{Narrative architecture}

The main concept of our infrastructure is the concept of the challenge. Challenge refers to an atomic part of the story, i.e, to the task that the player needs to accomplish to advance the game. The challenge is responsible for manipulating (creating, destroying or changing) the passive entities, through which the narrative is delivered to the player. In VGH, these entities include map overlays, ghosts (which are defined by their video and/or audio manifestations) and atmospheric background sound effects.

The lifecycle of the challenge is regulated by a set of controlling conditions, which consists of the precondition, the abort condition and the completion condition. Initially, each challenge is in the passive state. The passive challenge can never be completed unless it becomes active. A challenge is activated when its precondition is satisfied. An active challenge may become completed if a new event satisfies its completion condition. If the abort condition is satisfied it becomes passive again (see the state diagram in Fig. 1(a)). A challenge might change between active and passive states an arbitrary number of times, but it 
can only be completed once. A completed challenge can still be activated and deactivated and can manipulate its passive entities. This can be useful if the challenge controls a permanent part of the game world. In this case, completion of the challenge triggers the progress in the game but passive entities controlled by the challenge remain in the game and can enhance the further game experience.

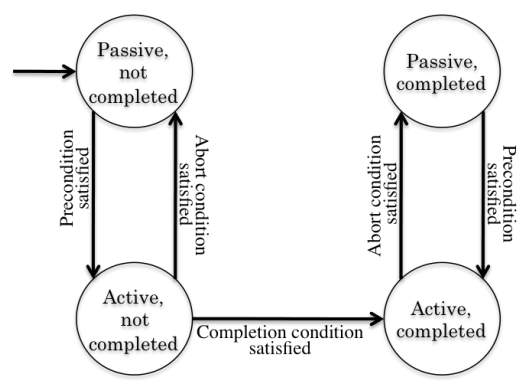

(a) Challenge state diagram

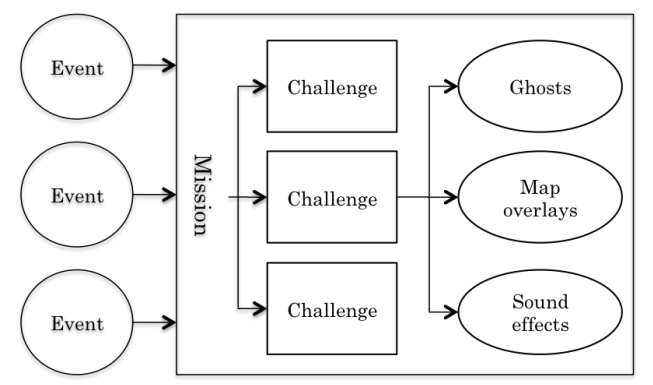

(b) Mission infrastructure

Fig. 1. Main components of the narrative architecture

Each change in the state of the challenge triggers the corresponding action. When the challenge is activated, its start action is triggered, deactivation triggers the stop action and completion triggers complete. These actions can be specified for each challenge separately and allow the challenge to adopt to its new state, for instance, by manipulating the managed passive entities. The change in the internal state of the challenge is not perceivable to the player but the change in passive entities can be perceived through the corresponding UI elements.

The challenges are notified about changes in the game state through events. Events are small objects that encapsulate relevant information about changes. Examples of events include receiving a new update about the relevant context changes (e.g., change of the location of the player), seeing or hearing the ghost manifestation or the completion of one of the challenges. This last type of events makes it possible to build a hierarchy of challenges by using completion of one of the challenges in the controlling conditions of another. For instance, in this way it is possible to chain the challenges, activating the next when the previous is completed. Another way to create a more complex structure is to define a master challenge that manages several smaller challenges and is completed when a certain number of these "child" challenges is completed. Some examples of such composite challenges are described in Section 5.

To bind all challenges together and to ensure delivery of events to each of them the challenges are encapsulated inside the mission component. This component handles all challenges that relate to the particular story. It is responsible for accumulating game events and delivering them to the challenges according to their internal state. When a new event is received the mission checks preconditions of passive challenges and activates those for which preconditions are satisfied. For active challenges the mission checks abort and completion conditions and changes the state of the challenges accordingly. Figure 1(b) shows 
the overall picture of the mission infrastructure. The arrows show how events are propagated through the mission to challenges, and how the challenges in response modify passive entities managed by mission.

In practice, there is no need to load all possible challenges into the infrastructure from the very start of the game. Instead we allow challenges to instantiate other challenges at the appropriate times. Therefore only a limited set of the initial challenges is instantiated first. Challenges are also allowed to unload themselves from the mission. After unloading they will no longer be notified about the new events, and the resources that they hold at this point can be deallocated. This strategy is particularly advantageous in the resource-constrained environment of the mobile device.

\section{Case study}

The mission that we used as a proof-of-concept for our narrative architecture is set in the surroundings of the medieval church of St. Audoen. The church lies within the old Viking city walls in Dublin and is surrounded by public parkland. There is a number of local stories associated with the place. In particular, the church has in its keeping the so-called 'Lucky Stone', a medieval tombstone, which is believed to possess magical qualities. Several ghosts from different times are believed to haunt the area including the Green Lady doomed to look eternally for her lost baby and the local gang member murdered in vicious street fights during the 18th century. Including these ghosts in the game allowed us to expand the focus of the mission from the Dublin Viking history to other parts of the city's past without breaking consistency of the story.

The mission unfolds as follows. When players launch the game, the icon on the map notifies them that the surroundings of St. Audoen's church are haunted. Once the player reaches the site they begin undertaking an investigation by searching for and locating evidence of paranormal activity. In this part of the game, spots of paranormal activity are randomly located around the site. The evidence that the player gathers at this point include pictures of Viking artifacts and 'ghostly' sounds. This gives the player a chance to get used to the game interfaces while at the same time getting immersed in the atmosphere of historical/paranormal investigation. Once the player gathers enough evidence of paranormal activity the new ghost appears. This is Olaf, a trapped Viking ghost who solicits the player to help him escape and thus provides the player with a motivating goal for the rest of the mission. At this point, Olaf also delivers the player's first explicit challenge commanding him to find the Lucky Stone. After finding the ghostly remnant of the Stone at the spot where it was originally located the player returns to Olaf to receive further instructions.

At this point the player is confronted with a narrative fork that contains a dramatic choice. By way of completing the final challenge, the player must choose between two routes and two approaches: take the easiest and quickest Southern route but at great risk to Olaf or take the longer and more dangerous Northern route and face impending doom. On both routes the player encounters 
other St. Audoen's ghosts, and Olaf tells their stories to the player. Both routes eventually lead the player to the same target: the garden where Olaf wants to return. Once the player reaches the garden he or she receives Olaf's thanks as well as a medieval coin as a token of Olaf's appreciation.

We implemented the described mission using the narrative architecture presented in Sec. 4. To capture the story we use 30 instances of 11 different challenges. Most of these challenges havea fairly simple structure. For example, there is a challenge "Play narrative once near ghost", which is activated when the player sees the visible ghost. The challenge then starts playing an audio file associated with that ghost and is complete when the playback is finished. Seven instances of this challenge are used in the game, each one parametrized with the particular ghost and the audio file. Two more interesting challenges, which we would like to draw attention to, are the transition between the atomic challenges part and the encounter with Olaf, and the branching point in the mission.

The activation condition for the ghost of Olaf is that the player found 3 out of 10 pieces of evidence of paranormal activity located in the region. Finding each piece of evidence constitutes a simple atomic challenge: its precondition is that the player is in the region and the completion condition is that the player captures the corresponding piece of evidence. In order to keep track of how many atomic challenges have been accomplished, a more complex challenge is created (see Fig. 2(a)). This challenge maintains an internal counter, which is increased each time when one of the atomic challenges is completed. The completion condition for this challenge is that this internal counter reaches the number of 3. When the challenge is complete it activates the new 'find Olaf' challenge. Also, the completion of this challenge is a signal for remaining atomic challenges to abort and to release their resources.

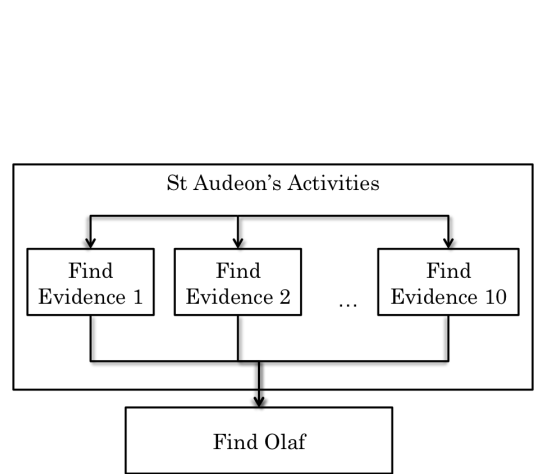

(a)

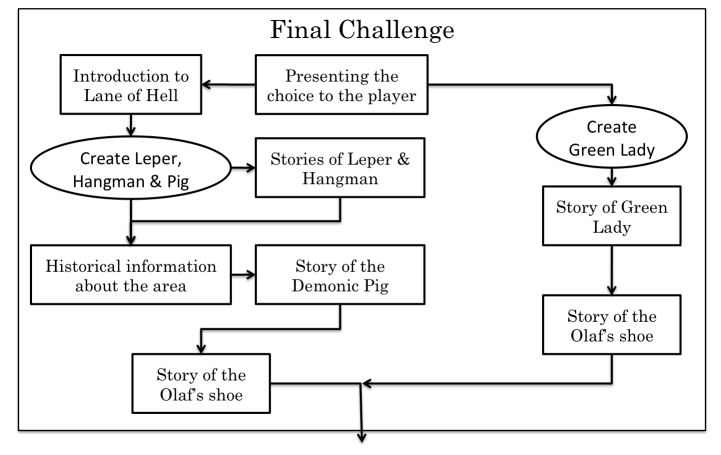

(b)

Fig. 2. Structure of some sample challenges

A more complicated challenge is used to manage the two branches of the game. The principal scheme of this challenge is presented in Fig. 2(b). Subchallenges that are managed by this challenge are represented as rectangular 
shapes while oval shapes mark the actions by which the superchallenge reacts in response to completion of the subchallenges. Subchallenges in this case are responsible for triggering pieces of narrative either at certain locations or when the player sees the visible ghost.

At the branching point the progression of the game is determined by which of the two challenges gets completed first. If the player follows the Northern route they will first encounter the Lane of Hell while on the Southern route the Green Lady will be met. Depending on which of the two the player reaches first, the remaining challenges of the Northern or the Southern route are activated while the challenge associated with the alternative branch is removed. Some challenges placed on the Northern route are optional. For example, passing the Lane of Hell the player can encounter some of the ghosts haunting the lane (the Leper and the Hangman) and learn their stories. However, if the player does not find them it is still possible to proceed to the subsequent parts of the game. Other pieces of narrative are critical for the consistence of the story and therefore mandatory.

\section{Discussion}

In Sec. 3 we stated the key requirements for the narrative supporting architecture for Viking Ghost Hunt. In this section we review our proposed architecture to determine the extent to which it meets these requirements.

Delivering location-specific (and, generally, context-specific) narrative is ensured by the ability of challenges to react to changes in the context through events. As challenges are capable of changing their state in response to the events, the delivered narrative can be context-specific. Non-linearity of the story is achieved by the fact that multiple challenges are allowed to be active simultaneously. In this case, the player's actions define which of the challenges will be accomplished and therefore which branches of the story will be unlocked next. The resource management is simplified by the fact that the resources are managed by each challenge autonomously. Together with "on-demand" loading of challenges, this ensures that only the necessary resources are allocated, and that the resources are deallocated as soon as they are no longer required.

It is not an easy task to evaluate the architecture in isolation from the game it was used in. To illustrate its applicability for a broader range of LBMGs, we examine how it can be used for supporting the five types of LBMGs identified in the survey undertaken by Kjeldskov and Paay [10]. The first type of games they mention is treasure hunt games with linear story. They can be represented as a sequence of challenges creating and loading the next one when completed. Jigsaw puzzles is the type of the games to which Viking Ghost Hunt most closely relates. Its defining characteristic is that the player collects seemingly random fragments of the narrative, which gradually combine into the bigger story. This type of narrative can be modeled by creating "branches" of challenges that all ultimately meet at one final point. As challenges from different branches are active simultaneously the user will always have the possibility to give up a certain branch to follow the other one (possibly even without noticing the fact). 


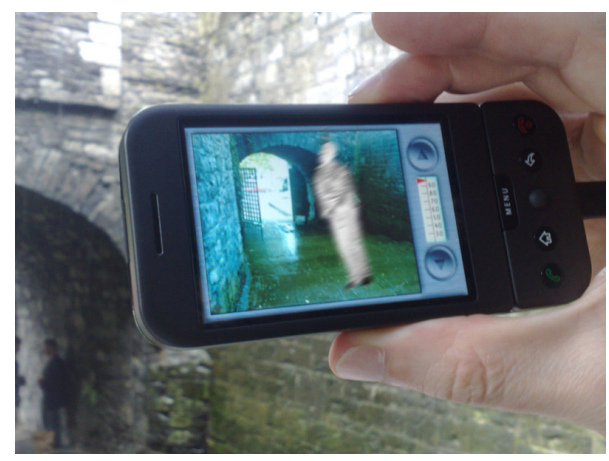

Fig. 3. Playing Viking Ghost Hunt: the ghost is seen on the screen

Domino games are similar to treasure hunt games except that the context in which the current narrative fragment is delivered suggests which of the several possible next fragments should be selected. This type of story can be supported by our framework since active challenges are notified about all relevant changes in the context. The context information will determine, which challenge is to be created and loaded next. Scrabble games offer to the player a highly interactive experience adopting the story to many events that are happening around the player. The example the authors give uses information from the Bluetooth profiles of the surrounding phones to modify the story. To create this kind of game with our framework it is necessary to introduce new types of game events to capture relevant information from the surroundings. Once this is accomplished it becomes possible to adapt the behavior of the challenges to the contextual information.In collecting butterflies type of games unrelated self-contained stories are linked to the locations. To support this type of games multiple challenges can be created, each using the player location in its controlling conditions.

\section{Conclusion}

In this paper we have presented an architecture to support location-based and, more broadly, context-sensitive interactive narrative. The architecture has been successfully applied in practice to develop a location-aware mobile game Viking Ghost Hunt for Android handsets. We have also discussed the capability of the architecture to support a wide range of LBMGs and interactive mobile guides.

The architecture is specifically designed to meet the requirements of the resource-constrained environment of the smartphone and can be applied to simplify the development of future mobile applications. More extensive evaluation including field user trials is reserved for future work.

\section{Acknowledgements}

We are grateful to the National Digital Research Center (NDRC) for funding the work described in this paper. 


\section{References}

1. Aarseth, E.: Quest games as post-narrative discourse. In: Narrative across media: The languages of storytelling. pp. 361-376. U. of Nebraska Press (2004)

2. Adams, E., Rollings, A.: Fundamentals of Game Design. Prentice Hall, Upper Saddle River, NJ (2006)

3. Benford, S., Crabtree, A., Flintham, M., Drozd, A., Anastasi, R., Paxton, M., Tandavanitj, N., Adams, M., Row-Farr, J.: Can you see me now? ACM Trans. Comput.-Hum. Interact. 13(1), 100-133 (2006)

4. Björk, S., Falk, J., Hansson, R., Ljungstr, P.: Pirates! Using the physical world as a game board. In: Interact 2001:8th IFIP TC.13 Conf. on HCI. pp. 9-13 (2001)

5. Brunnberg, L., Juhlin, O., Gustafsson, A.: Games for passengers: accounting for motion in location-based applications. In: FDG '09: Proc. of the 4th Int. Conf. on Foundations of Digital Games. pp. 26-33 (2009)

6. Cheverst, K., Davies, N., Mitchell, K., Friday, A., Efstratiou, C.: Developing a context-aware electronic tourist guide: some issues and experiences. In: CHI '00: Proc. of the SIGCHI Conf. on Human Factors in Comp. Systems. pp. 17-24. ACM, New York, NY, USA (2000)

7. Dow, S., Lee, J., Oezbek, C., MacIntyre, B., Bolter, J.D., Gandy, M.: Exploring spatial narratives and mixed reality experiences in Oakland Cemetery. In: Advances in Computer Entertainment Technology. pp. 51-60. ACM (2005)

8. Howard, J.: Quests : Design, theory, and history in games and narratives. A.K. Peters, Wellesley, Massachusetts (2008)

9. Juul, J.: Half-Real: Video Games Between Real Rules and Fictional Worlds. MIT Press, Cambridge, Massachusetts (2005)

10. Kjeldskov, J., Paay, J.: Augmenting the city with fiction: Fictional requirements for mobile guides. In: Mobile Interaction with the Real World 2007/5th Workshop on HCI in Mobile Guides Singapore. pp. 41-45 (2007)

11. LeBlanc, M.: Feedback systems and the dramatic structure of competition. In: Game Developers Conference (1999)

12. Malaka, R., Schneider, K., Kretschmer, U.: Stage-based augmented edutainment. In: Smart Graphics: 4th International Symposium. pp. 54-65 (2004)

13. Mateas, M., Stern, A.: Structuring content in the facade interactive drama architecture. In: Proc. of the 1st Art. Intelligence and Interact. Dig. Entertainment Conf. pp. 93-98 (2005)

14. Murray, J.H.: Hamlet on the holodeck : The future of narrative in cyberspace. Free Press, New York (1997)

15. Nisi, V., Oakley, I., Haahr, M.: Location-aware multimedia stories: Bringing together real and virtual spaces. In: ARTECH Conf. on Dig. Arts. pp. 72-81 (2008)

16. Paay, J., Kjeldskov, J., Christensen, A., Ibsen, A., Jensen, D., Nielsen, G., Vutborg, R.: Location-based storytelling in the urban environment. In: OZCHI '08: Proc. of the 20th Australasian Conf. on CHI. pp. 122-129. ACM (2008)

17. Roberts, D.L., Cantino, A.S., Jr., C.L.I.: Player autonomy versus designer intent: A case study of interactive tour guides. In: AIIDE. pp. 95-97 (2007)

18. Salen, K., Zimmerman, E.: Rules of play: game design fundamentals. MIT Press, Cambridge, Massachusetts (2004)

19. Tosca, S.: The quest problem in computer games. In: Proc. of The Technologies for Interact. Dig. Storytelling and Entertainment Conf. (TIDSE). Darmstadt (2003) 